\title{
Domino liver transplantation using livers from patients with familial amyloidotic polyneuropathy
}

Pr Alexandre José Linhares Furtado

\begin{abstract}
Transplantation of livers from patients with familial amyloidotic polyneuropathy undergoing orthotopic liver transplantation is based on the normal functions of these livers, except for the production of an abnormal protein that causes the disease, which takes more than 20 years to become clinically apparent. Given the shortage of cadaver grafts, domino liver transplantation has the potential to increase the offer to some selected patients. The known experience amounts to little more than 80 cases. Domino liver transplantation has been a safe procedure with all the advantages of living donor grafts. So far, the disease has not shown up in any recipient, including the longest surviving patient (4 years). Particularly in countries where the disease is common, the potential to increase graft offer may be significantly augmented through cooperation between centers and better exploitation of technical possibilities. The combination of split and domino has been reported six times and may provide three to six transplants from only one cadaver graft. Curr Opin Organ Transplant 2000, 5:69-73 @ 2000 Lippincott Williams \& Wilkins, Inc.
\end{abstract}

Department of Transplantation, Hospitals of the University of Coimbra, Portugal Correspondence to Pr. Alexandre José Linhares Furtado, Departamento de Transplantação, Hospitais da Universidade de Coimbra, Av. Bissaya Barreto, 3049 Coimbra Codex, Portugal

Current Opinion in Organ Transplantation 2000, 5:69-73

Abbreviations

DLTx domino liver transplantation

TTR transthyretin

ISSN 1087-2418 @ 2000 Lippincott Williams \& Wilkins, Inc.
Domino organ transplantation was probably mentioned for the first time to describe the transplantation of a heart graft retrieved from a patient undergoing a heartlung transplantation. The heart of this patient was normal and was transplanted into a second patient with end-stage cardiac dysfunction [1]. A similar procedure was accomplished with the liver in 1991 [2], although in this case the graft was procured from a liver recipient who suffered brain death.

On October 26, 1995, for the first time, the whole liver of a living patient with familial amyloidotic polyneuropathy (FAP) receiving a cadaver graft was transplanted into a man with isolated liver metastasis from a sigmoid cancer. After our report of the first cases of this "sequential liver transplantation" $[3,4]$, the procedure was soon accepted $[5,6,7 \bullet, 8 \bullet \bullet]$ and became better known as domino liver transplantation (DLTx).

Familial amyloidotic polyneuropathy is an autosomal hereditary dominant disorder (linked to chromosome 18) whose biochemical basis depends on the production, almost exclusively by the liver, of an abnormal transthyretin (TTR). There are several variants, the most common being the TTR Met 30, in which the normal valine is replaced by methionine. This mutant is typical of all cases of the disease in Portugal, the country with the highest incidence of FAP and where the disease was first scientifically described [9]. Besides Portugal, FAP patients are particularly concentrated in Sweden and Japan, but rare cases are found almost all over the world. Although this review is restricted to the use of livers from FAP Met 30 patients, this domino procedure will be cited simply as DLTx.

Liver transplantation was first used to treat FAP in 1990 and remains the only hope for these patients [10-13]. A total of $368 \mathrm{FAP}$ patients who had undergone transplants had been reported to the FAP World Transplantation Registry as of June 1999 [14], but we believe that more than 500 have already undergone transplant worldwide. The livers of FAP patients are anatomically normal, having only one functional abnormality-the production of the abnormal TTR. Thus it is appealing to use the livers of FAP patients as living donor grafts for selected patients with other liver diseases, under well-defined conditions. Although the contribution of procured grafts from cadavers of patients who have previously undergone transplants has been and is expected to remain 
extremely small [15], DLTx using organs from FAP patients may have the potential to significantly decrease organ shortage, mainly in countries where this disease is commonly found.

\section{Rationale}

Even among the population groups with the earliest onset of symptoms, FAP never becomes a clinical entity before the patient is 20 years old (later for most patients). The progression to death takes 10 to 15 years and the disease usually remains tolerable for the first half of this period.

It must be assumed that FAP may develop in nongenetically affected recipients of DLTx. Indeed, the abnormal protein appears in the blood of the recipients shortly after transplantation. It is highly probable that the disease, if it develops, will follow the same course as in the genetically affected patients. This means that more than 25 years would elapse without relevant symptoms. So, for a 50-year-old patient, with a nonresectable malignancy of the liver, the opportunity that he or she otherwise would not have (to receive a transplant from the waiting list for cadaver grafts) seems an adequate indication. This was our original proposal for the strategy of DLTx [4] and remains the most unquestionable indication. Indeed, the offer of a life expectancy of 25 years or more for such a patient would be a blessing, provided the transplant itself will remain functioning throughout this period and the tumor does not recur. The use of FAP liver grafts bears the great advantages of any living donor graft: excellent preharvesting hemodynamic conditions, few minutes of warm ischemia before University of Wisconsin (UW) hypothermic perfusion (average 3 minutes, in our experience), very short total ischemia time, and excellent initial function.

\section{Ethical principles}

The most important ethical principle concerns the informed consent of the domino recipient, including the need to underscore that the patient may develop paramyloidosis if he or she survives long enough. The patient must be assured that, should the FAP appear, he or she would have an opportunity to receive a non-FAP cadaver graft. According to Portuguese law and to our guiding ethical principles, there is no need to inform the FAP donor about the use of his or her liver in another patient. However, all our patients have previously been informed about the procedure. They were happy to know that their organs could be of some benefit to other patients, whom they met later in the ward. Also, the FAP patients must be assured that the technical demands of the hepatectomy for a domino will not impose on them any unusual risks.

\section{Indications}

Our original aim was to use the domino strategy for nonresectable hepatocellular cancers or neuroendocrine tumors (excluding the first patient, who had liver metastasis from a sigmoid cancer). However, the pressure from a long waiting list with other pathologies and high risk patients of over 50 years led us to offer the domino technique in selected noncancer cases, mainly to patients with hepatitis B and C or alcoholic cirrhosis.

A consensus report of the Hospital of the University of Coimbra Ethical Committee in 1994 supported our trend toward a flexible attitude in view of some of these patients. Thus, in December 1996, we implanted an FAP liver graft into a patient with alcoholic cirrhosis. Thus far we have performed 13 DLTxs with FAP grafts in patients without malignancy. In two extremely critical situations, a third cadaver transplant was unanimously excluded but a domino as a third transplant in these two young patients was promptly accepted (one of them, a 28-year-old-man, is alive and well; the other died of continuous sepsis).

The indications for domino procedures must take into account many factors, including age, risks, probability of recurrences, global prognosis, and priorities in the candidate lists. In the special case of Portugal, all the waiting lists in the four liver transplant centers include a large number of FAP patients eagerly awaiting transplantation, whose deteriorating condition may soon preclude the only hope of halting the progression to death. A sort of "competition" between FAP and non-FAP candidates exists and the domino may minimize this dilemma in a nonnegligible number of cases. The FAP cases in our transplant experience represent $33 \%$ of all the patients undergoing transplantation; this percentage is even higher in the other transplant centers of Portugal. In our total experience of 27 DLTxs (Hospital of the University of Coimbra), the dominos amount to $9 \%$ of all patients undergoing transplantation.

The patient survival rate for orthotopic liver transplantation at 10 years in European centers is about $60 \%$. The survival rate at 30 years is not yet known, but it seems reasonable to presume that by that time, most patients who received a domino FAP graft at the age of 50 will be dead from causes other than paramyloidosis (including the harmful effects of immunosuppression).

\section{Main technical points and contribution to increased graft offer}

Hepatectomy in the FAP patient must be carried out under conditions such that the liver may be safely used as a graft in another patient. The hepatectomy must include the retrohepatic vena cava and, as a consequence, the use of an external venovenous bypass has been the rule in our experience, even if the FAP liver is to be implanted as a piggy-back. The FAP patients are hemodynamically rather fragile [16-19] and have no 
portal collateral circulation; thus, in our view, the venovenous bypass affords total safety, although there are several reports $[8 \bullet \bullet, 20]$ of procedures performed without venous bypass and no problems were reported.

We think that the vena cava-preserving hepatectomy in FAP is inadequate for using the liver as a whole graft. Despite our experience of more than 200 hepatectomies with preservation of the vena cava in the recipient (including most FAP patients), the procedure is substantially more difficult than hepatectomy including the vena cava. The difficulties would be greater if the liver were used as a graft; the quality of the graft could be jeopardized (some accessory hepatic veins must be ligated) and its implantation would be enormously more difficult, if not impossible. The difficulties and the inherent risks would be higher for the FAP patient.

In some hepatectomies for DLTx, a transdiaphragmatic dissection of the vena cava may be advisable to keep good segments of that vein both with the graft and with the FAP patient. As we noted in our first reports [3,4], the division of the diaphragmatic fibrotic ring around the vena cava requires particular care, but it is a safe step for an experienced surgeon. However, dividing the suprahepatic segment of the vena cava just flat to the liver may spare this step and would not preclude an adequate implantation. In this case and if a classic implantation is performed, the shortness or even the absence of the segment of the suprahepatic vena cava in the graft may be overcome with a venous graft extension, on the back table. This vena cava graft may be obtained from the infrahepatic vena cava of the same graft or from a cadaver. We have performed this venoplasty three times, without complications.

The division of the hilar structures in the FAP patient must respect the best conditions for excellent anastomosis to the corresponding structures of the cadaver graft that the patient is going to receive. The surgeon should examine this graft before surgery to perfect the procedure and allow for greater technical flexibility.

The domino graft may be implanted in the classic fashion or as a piggy-back. We consider the classic technique more appropriate for patients with tumors, especially when they are sited not far from the vena cava; for benign cases we always prefer the piggy-back technique without external venovenous bypass.

The use of DLTx has, so far, contributed 75 grafts in the seven centers (Table 1) that replied to a short questionnaire aimed at updating the international experience on this subject. However, if we add some known cases from centers that did not reply, surely more than 80
Table 1. Use of domino liver transplantation in seven centers

\begin{tabular}{lccc}
\hline Centers & $\begin{array}{c}\text { Dominos } \\
\text { performed, } n\end{array}$ & $\begin{array}{c}\text { Cases of } \\
\text { malignant } \\
\text { pathology, } n\end{array}$ & $\begin{array}{c}\text { Benign } \\
\text { causes, } n\end{array}$ \\
\hline Heidelberg, Germany & 1 & 1 & 0 \\
Ghent, Belgium & 2 & 0 & 2 \\
Hudding, Sweden & 6 & 6 & 0 \\
St. Antonio, Porto & 7 & 5 & 2 \\
S. Paulo, Brasil & 15 & 11 & 4 \\
Villejuif, France & $17^{\star}$ & 16 & 1 \\
Coimbra, Portugal & $27^{+}$ & 14 & 13 \\
Total & 75 & 53 & 22 \\
\hline
\end{tabular}

*Three of these procedures were split plus domino. ${ }^{\dagger}$ Two of these procedures were split plus domino.

cases have been performed. This contribution to increased graft offer is small when compared with the number of liver transplants performed in each center. Three ways of increasing that contribution may be envisaged: broadening the field of indications; combining the domino with splitting; and intensifying cooperation between centers. The combination of all three attitudes would provide the best chances of optimum sharing.

At the moment FAP grafts have been used mainly in benign cases and malignant liver pathologies, but presumably the number of benign cases will increase in the future, although paramyloidosis could unexpectedly show up early in a single or in several dominotransplanted patients. This has not happened so far in any patient, including one patient followed up for 4 years and three patients followed up for 3 years at the Hospital of the University of Coimbra. The clinical assessment and neurologic evaluation of these patients continue to show no signs of the disease. In three of these domino-transplanted patients, cutaneous biopsies were performed 2.5 years after transplantation and no amyloid deposits were found. This leads us to plan new biopsies no sooner than 5 years after the domino transplants.

The combination of the split with the domino has been accomplished at least six times $[21 \bullet \bullet, 22,23]$, allowing three transplants (Fig. 1), starting with only one cadaver graft. In one of these combinations [23], the split was done in situ in the FAP patient.

The furthest extension of this combined procedure will be splitting a cadaver graft, using the two splits in two FAP patients and then splitting the two FAP livers to perform four dominos. Thus, starting with one cadaver graft, six transplants would be accomplished (Fig. 2). However, it is hardly conceivable that any transplant center would meet the logistics (staff and facilities) required for such a long, difficult task, at least routinely. Therefore the combination of split and domino may 
Figure 1. Two strategies for three transplants, starting from one cadaver graft

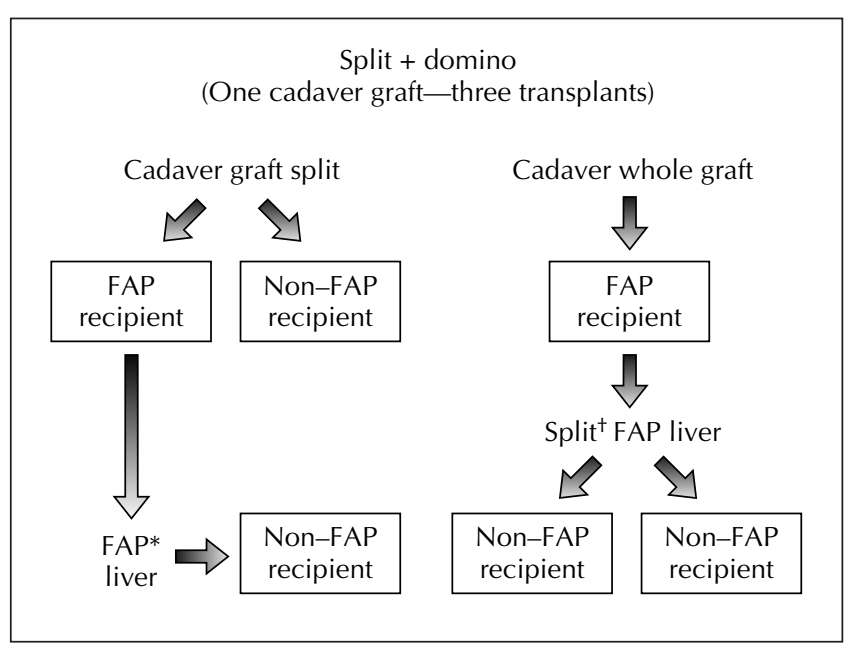

${ }^{*}$ If split, 4 transplants may be performed. ${ }^{+}$In the bench or in situ. FAP, familial amyloidotic polyneuropathy.

provide three to six transplants, but so far only three transplants have been achieved in each procedure already reported.

In our view, the best chances for optimum exploitation of organ offering and sharing when using the domino procedure lie in cooperation between centers. In a small center like ours (with limited staff and facilities) the combination of split and domino is an exhausting achievement. But splitting the cadaver graft and locally using one of the splits while sending the other to another center to receive from it, hours later, an FAP graft, is a much more acceptable challenge. This cooperation will substantially diminish the stress on the

Figure 2. Strategy for six transplants, starting from one cadaver graft

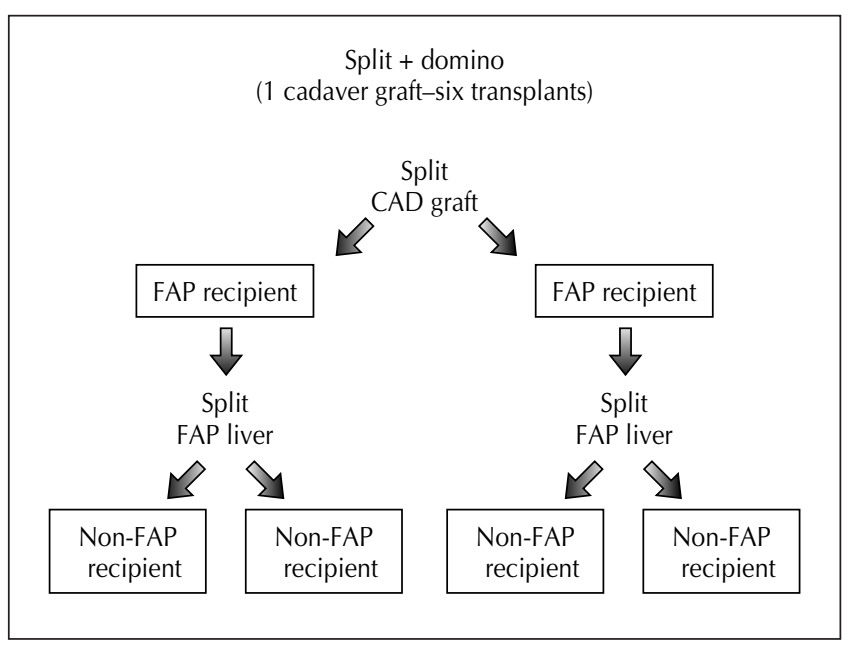

FAP, familial amyloidotic polyneuropathy. staff and, in our case, may allow the reuse of the same operating room. With good coordination, it allows better selection of the recipients, keeps the ischemia times shorter, and may increase the opportunities for a triple transplant from a single cadaver graft. This cooperation may increase the number of transplants and may become a significant contribution in countries with an important number of FAP candidates awaiting liver transplantation, along with the usual non-FAP candidates.

\section{International experience}

So far, only a limited number of DLTx cases have been reported. From the restricted questionnaire that we have previously mentioned, it may be stated that all centers followed the same general guiding principles for the 75 dominos reported, most of them performed on patients older than 50 years with malignancies. We are sure that the procedure has been performed in more than 80 cases. Repeated neurologic assessments and biopsies have been performed showing no signs of FAP disease or deposits of amyloid in skin and nerves at 2.5 years after transplantation (the longest follow-up, at the Hospital of the University of Coimbra). There have been no deaths. The incidence of relevant complications in the FAP patients, ascribable to the specific technical problems of the domino procedures, was very small (only four reported and some doubtfully related to the technical peculiarities of the domino procedure).

\section{Conclusions}

Domino liver transplantation with FAP livers is an innovative strategy that is contributing to increased liver graft offer. It is indicated in patients whose age or prognosis would cast doubts on the use of a cadaver graft or would delay or even preclude their receiving transplants from waiting lists with higher priorities. The procedure has been shown to be safe both for the FAP donor and the recipient and it has all the advantages of living donor grafts. In communities where the disease is commonly found, the contribution for increasing the number of transplants may become significant. The combination of split and domino may contribute to a further increase in the number of transplants. Good cooperation between different centers may overcome the difficulties for optimum use of this strategy. It is expected that the appearance of FAP disease in the domino recipient will not occur earlier than than 20 years after DLTx. Periodic evaluation of DLTx recipients is required.

\section{Acknowledgments}

The author would like to thank all who contributed to this review, particularly the chairmen of the transplant centers that provided information on their experience of DLTx, Prof. H. Bismuth for his personal communication about the splitdomino technique, and Prof. Morell and Prof. Caetano Pereira for the sharing of FAP grafts. 


\section{References and recommended reading}

Papers of particular interest, published within the annual period of review, have been highlighted as:

- Of special interest

.. Of outstanding interest

1 Klepetko W, Wollenek G, Laczkovics A, Laufer G, Wolner El: Domino transplantation of heart-lung and heart: an approach to overcome the scarcity of donor organs. J Heart Lung Transplant 1991, 10 (Part 1):129-131.

2 Moreno EG, Garcia GI, Gonzalez-Pinto I, Gómez SR, Loinaz SC: Successful reuse of a liver graft. Br J Surg 1991, 78:813-814.

3 Furtado AJL: Sequential liver transplantation. Oral communication in: Second International Workshop on Liver Transplantation in Familial Amyloidotic Polyneuropathy, Lisbon, 1995.

4 Furtado A, Tomé L, Oliveira FJ, Furtado E, Viana J, Perdigoto R: Sequential liver transplantation. Transplant Proc 1997, 29:467-468.

5 Hesse U, Troisi R, Mortier E, Decruyenaere J, de Hemptinne B: Sequential orthotopic liver transplantation: domino transplantation. Chirurg 1997, 68:1011-1013.

6 Hemming AW, Catral MS, Shari RS, Greyg PD, Lilly LB, Ashby P, Levy GA: Domino liver transplantation for familial amyloid polyneuropathy. Liver Transplant Surg 1998, 4:236-238.

7 Schmidt H H-J, Nashan B, Propsting MJ, Nakazato M, Flemming Peer,

- Kubicka S, et al.: Familial amyloidotic polyneuropathy: domino liver transplantation. J Hepatol 1999, 30:293-298.

This is a detailed account of two dominos with broad information focusing on biochemical aspects.

8 Azoulay D, Samuel D, Castaing D, Adam R, Adams D, Said G, Bismuth H:

- Domino liver transplants for metabolic disorders: experience with familial amyloidotic polyneuropathy. J Am Coll Surg 1999, 189:584-593.

This is a detailed report of a very important experience.

9 Andrade C: Clinique de la Paramyloidose du type portugais. Acta Neuropathol 1963, suppl II:3-11.

10 Holmgren G, Steen L, Ekstedt J, Groth CG, Ericzon BG, Eriksson S, et al.: Biochemical effect of liver transplantation in two Swedish patients with familial amyloid polyneuropathy (FAP-met 30). Clin Genet 1991, 40:242-246.

11 Holmgren G, Ericzon B-G, Groth C-G, Steen L, Suhr O, Andersen O, et al.: Clinical improvement and amyloid regression after liver transplantation in hereditary transthyretin amyloidosis. Lancet 1993, 341:1113-1116.
12 Ericzon BG, Suhr O, Broome U, Homgren G, Duraj F, Eleborg G, et al.: Liver transplantation halts the progress of familial amyloidotic polyneuropathy. Transplant Proc 1995, 27:1233.

13 Monteiro E, Perdigoto, R, Furtado AL: Liver transplantation for familial amyloid polyneuropathy. Hepatogastroenterolgy 1998, 45:1375-1380.

14 Familial Amyloidotic Polyneuropathy World Transplant Register. 1999 Update. Stockholm, Sweden: Karolinska Institute; 1999.

15 Lowell JA, Taranto SE, Singer GG, Miller SB, Ghalib R, Caldwell C, et al.: Transplant recipients as organ donors: the domino transplant. Transplant Proc 1997, 29:3392-3393.

16 Suhr O, Wiklund U, Eleborg L, Ando Y, Backman C, Birgersdotter V, et al.: Impact of autonomic neuropathy on circulatory instability during liver transplantation for familial amyloidotic polyneuropathy. Transplantation 1997, 63:675-679.

17 Eleborg L, Suhr O, Gunnarsson L: Familial amyloidotic polyneuropathy (FAP): cardiac and circulatory function during anesthesia for liver transplantation. Amyloid 1997, 4:24-32.

18 Viana JS, Bento C, Vieira H, Neves S, Seco C, Elvas L, et al. Haemodynamics during liver transplantation in familial amyloidotic polyneuropathy: study of the intraoperative cardiocirculatory data of 50 patients. Rev Port Cardiol 1999, 18:689-697.

19 Viana JS, Furtado AL: Evaluation of the incidence and origin of hypotension during liver transplantation for familial amyloidosis. Br J Anaesth 1999, 82(suppl):41-42.

20 Hemming AW, Cattral MS, Greig PD, Lilly LB, Ashby P, Levy GA: Domino liver transplantation for familial amyloid polyneuropathy: optimal use of a scarce resource. Transplant Proc 1999, 31:515.

21 Furtado L, Oliveira F, Furtado E, Geraldes B, Reis A, Viana J, et al.:

-. Maximum sharing of cadaver liver grafts: composite split and domino liver transplantation. Liver Transplant Surg 1999, 5:157-158.

This is the first report of combined strategies calling attention to important technical details.

22 Stangou AJ, Heaton ND, Rela M, Pepys MB, Hawkins PN, Williams R: Domino hepatic transplantation using the liver from a patient with familial amyloid polyneuropathy. Transplantation 1999, 65:1496-1498.

23 Azoulay D, Castaing D, Adam R, Mimoz O, Bismuth H: Transplantation of three adult patients with one cadaveric graft: wait or innovate. Liver Transpl 2000, 6:239-240 